\title{
Spatial localization of a goal: Beacon homing and landmark piloting by rats on a radial maze
}

\author{
LAURA A. HOGARTH, WILLIAM A. ROBERTS, SHELLEY ROBERTS, and BENJAMIN ABROMS \\ University of Western Ontario, London, Ontario, Canada
}

\begin{abstract}
We performed six experiments in order to examine the ability of rats to use moving beacons and landmarks as cues to the location of reward on an eight-arm radial maze. In Experiments 1-4, the cues and goals were moved before each trial, and groups in which a single beacon was placed on the rewarded arm, a single landmark indicated that reward was on the arm immediately to the left of a landmark, or two landmarks were placed on each side of the reward arm were compared. The rats rapidly learned to track the reward in the beacon condition, failed to find the reward sooner than chance expectation with a single landmark, and did only slightly better than chance with two landmarks. In Experiments 5 and 6 , the rats were trained in five trials per day, with the landmark and goal locations constant over daily rewarded trials, and in two extinction trials that were inserted among the rewarded trials. The rats found the goal arm at substantially better than chance expectancy with both one and two landmarks. Our results, in agreement with data from recent swimming pool experiments (A. D. L. Roberts \& Pearce, 1998), show that rats will use the relationship between moving landmarks and a goal in order to find reward.
\end{abstract}

Animals show a considerable ability to navigate accurately over spatial terrains to find food, water, mates, and their home bases. The mechanisms of spatial cognition used by animals are undoubtedly multiple and interactive (W. A. Roberts, 1998a, 1998b). Thus, the mechanisms used may vary between situations and species and may change as an organism becomes increasingly familiar with its spatial environment. Spatial cognition mechanisms may generally be divided into internal, egocentric mechanisms and external, allocentric mechanisms.

Egocentric mechanisms include path integration or dead reckoning. In the absence of vision, organisms as diverse as insects and humans can travel a path that has many turns and still take a direct route back to a position very near the start of their travel (Gallistel, 1990; Loomis et al., 1993). Cues provided by vestibular and kinesthetic receptors allow an organism to maintain a homeward vector even though it may make several turns and travel varying distances in several directions (Etienne, Berlie, Georgakopoulos, \& Maurer, 1998). Another internal mechanism is the use of learned response rules. For example, rats may learn to always turn left or right at the choice point in a Tmaze or may learn to alternate turns on successive choices.

External cues may be used in several ways to find spatial locations. Cheng (1986) trained rats to find buried food in the corner of a dimly illuminated rectangular arena. Although the rats frequently dug in the exact location of

Support for this research was provided by a research grant from the Natural Sciences and Engineering Research Council of Canada. Experiments $1-5$ were reported in a master's thesis submitted by L. A. Hogarth to the University of Western Ontario. Correspondence should be addressed to W. A. Roberts, Department of Psychology, University of Western Ontario, London, ON, N6A 5C2 Canada (e-mail: roberts@) julian.uwo.ca). the food, Cheng observed that they commonly made errors by digging in the corner diagonally opposite to the one containing food. He concluded that the rats were using the geometric framework of the arena as their main cue to the location of food. Thus, the rats had learned to dig in the corner with a long wall on the left and a short wall on the right. Because the diagonally opposite corner also had these characteristics, it was not surprising that the rats frequently erred by digging there.

Although animals may use geometric frames to specify spatial location when they are available, in many situations in the natural world obvious frames may not be discernable. In this case, animals may use beacons or landmarks to locate salient places. One important mechanism is beacon homing. If a prominent object, such as a tree or rock, is near a food location, an animal needs only to navigate toward that object or beacon in order to find the goal. In the laboratory, an animal might learn to always enter the arm of a maze that points toward the door of the test room in order to obtain reward.

In many cases, however, animals appear to be able to repeatedly find locations in space where no beacon is located. A classic example is that of rats that have learned to find a submerged platform while swimming in a tank filled with opaque water (Morris, 1981). Although the platform is located below a homogeneous surface, the rats learn to swim precisely to that location, even when they start from different positions along the side of the tank. Observations such as this have led theorists to suggest that animals are able to find a location in space by computing the distance and direction of the location from multiple landmarks (Cheng, 1989, 1994; Poucet, 1993; Suzuki, Augerinos, \& Black, 1980).

One way in which the ability of animals to use landmarks relationally can be studied is to move the landmark 
around in space from one trial to the next. In this case, the relationship between the landmark and the goal is the only reliable cue to the location of the goal. Any other objects (potential beacons) near the goal on one trial will be unreliable on subsequent trials. Biegler and Morris (1993, 1996) studied the ability of rats to find buried food in an arena when the food was always located at a fixed distance and in a fixed direction from a landmark, relative to a polarizing background cue. Although the rats learned to search near the landmark, they showed little ability to find the precise location of food relative to the landmark. Biegler and Morris (1993) concluded that animals may be prepared to use the rule of thumb, "if it moves, don't use it as a landmark" (p. 633). On the other hand, A. D. L. Roberts and Pearce (1998) found quite opposite results with rats tested in a swimming tank. When the rats had to find a submerged platform that was at a fixed distance and in a fixed direction from a landmark that was moved from session to session, they found the platform faster than did a control group for which the position of the landmark was irrelevant to the location of the platform. Surprisingly, they also found that the rats located the platform better when the landmark moved than when the landmark was at a fixed position. Thus, the findings of Biegler and Morris and those of A. D. L. Roberts and Pearce suggest very different conclusions about the ability of rats to track a goal relative to a landmark whose position changes.

In the experiments reported here, a new procedure was used to examine the ability of rats to locate a goal that has a specific relationship to a landmark. The rats were studied on an eight-arm radial maze, and therefore there were eight different possible goal locations. One arm of the maze was made visually distinctive by placing a white or black-and-white striped cover over it (Experiments 1 and 3-6) or by placing a white disk at the end of one arm (Experiment 2), rather than by placing large objects in the extramaze environment. The goal arm that contained food was either the arm that contained the cover (beacon homing condition) or an arm immediately adjacent to it (landmark piloting condition). Because the positions of the landmark and goal changed randomly from trial to trial, only the landmark was a valid indicator of the goal arm. The use of a moving landmark allowed the examination of Biegler and Morris's (1993) contention that rats will not use a moving object as a landmark. In Experiments 1-3, we examined rats' ability to learn to use a single distinctive arm as a beacon or landmark. In Experiments 4-6, we tested conditions in which the goal was placed in a fixed relationship to two landmarks. In Experiments 5 and 6 , the testing procedures used by A. D. L. Roberts and Pearce (1998) in their water tank experiments were adapted to the radial maze in an attempt to find improved landmark piloting.

\section{EXPERIMENT 1}

The experimental apparatus was a black eight-arm radial maze. The beacon or landmark was a white arm cover that could be placed on any one of the eight arms of the maze. The arm chosen to be the white arm was changed randomly from trial to trial. Three groups of rats were trained on the maze, a beacon homing group, a landmark piloting group, and a control group. For the rats in the beacon homing group, a food reward was always placed at the end of the white arm. For the rats in the landmark piloting group, the food was always placed on the arm immediately to the left of the white arm. For the rats in the control group, the reward arm was chosen randomly and independently of the arm chosen to contain the white cover. The questions of interest were whether animals in the beacon homing group could learn to approach the white arm as a signal for food and whether animals in the landmark piloting group could learn to use the relationship between the landmark arm and the rewarded arm to find the reward.

\section{Method}

Subjects. Twelve experimentally naive male Long-Evans hooded rats were tested. They were housed in shoebox cages in a room illuminated from 7:00 a.m. to 7:00 p.m. For the duration of the experiment, the rats had constant access to water and were kept on a growth curve that maintained their weight at approximately $85 \%$ of their free-feeding weight.

Apparatus. An open eight-arm radial maze painted flat black was used. The octagonal center was $35.5 \mathrm{~cm}$ in diameter, and each arm was $72.5 \mathrm{~cm}$ long and $8.75 \mathrm{~cm}$ wide. A food cup, which was $3 \mathrm{~cm}$ in diameter and $1 \mathrm{~cm}$ deep, was located $2.5 \mathrm{~cm}$ from the end of each arm. A metal arm cover, painted flat white, served as the landmark. The cover sat flush to the surface of the arm and had the same smooth texture as the arm; it could easily be moved from arm to arm and extended $66.5 \mathrm{~cm}$ from the central platform to a location just before the food cup. The maze contained no walls, was elevated $60 \mathrm{~cm}$ above the floor on wooden legs, and was situated in a $3.5 \times$ $2.5 \mathrm{~m}$ room that contained a number of extramaze cues, including a chair, a shelf, a plant hanger, some hanging wires, and an audio speaker.

Procedure. During a preliminary training period, the rats were placed on the maze and were allowed to explore it and find Sun-Life brand miniature chocolate chips on the arms. Formal testing began once all of the rats could travel down all of the maze arms and retrieve one chocolate chip from each food cup.

Four rats were assigned randomly to each of three groups, the beacon homing group, the landmark piloting group, and the control group. On each trial for each rat, the white cover was placed on a randomly chosen arm, and only one arm was baited with a chocolate chip. The placement of the reward in relation to the landmark varied among groups. The reward was always placed at the end of the white arm for the rats in the beacon homing group. In the landmark piloting group, the reward was always placed on the arm adjacent to and to the left of the landmark arm (as viewed from the center of the maze). A different sequence of random landmark locations was used for each rat. In the case of the control group, random sequences of rewarded arms were used to determine placement of the reward on each trial. Thus, the relationship between the white arm and reward was unpredictable for the control group.

The rats were tested on two trials per day between the hours of 11:00 a.m. and 2:00 p.m. Each rat was placed on the central platform of the maze, facing in a randomly chosen direction on each trial. It was allowed to enter arms freely on the maze until it entered the reward arm and ate the reward. An arm entry was recorded manually each time a rat's back feet left the central platform. Once each rat had located the goal and returned to the central platform, it was 
removed from the maze and returned to its home cage. Each rat within a group received its first trial of a session before a second round of trials was run. The intertrial interval was about $10 \mathrm{~min}$. Testing lasted for 15 sessions or 30 trials.

\section{Results}

All statistical tests were evaluated using an alpha level of .05. The degrees of freedom for trial effects and interactions in analyses of variance (ANOVAs) were adjusted using the Greenhouse-Geisser epsilon correction factor (Lewis, 1993).

A rat's ability to find the location of the reward arm was measured by the number of arm entries it made up to the time the reward was found. Thus, perfect performance would be a score of one arm entry. The data collected from the 30 trials were averaged into 10 blocks of 3 trials each. The mean number of arm entries made by each group at each of the 10 blocks is presented in Figure 1. A groups $\times$ blocks of trials ANOVA performed on arm entries revealed a significant difference between groups $[F(2,9)=18.82]$, a significant drop across blocks $[F(2,18)=6.02]$, and a significant groups $\times$ blocks interaction $[F(4,18)=7.20]$.

These data were divided into three stages of learning for further analysis. During the first stage of learning (Trial Blocks 1-3), the mean arm entries required by the control group, beacon homing group, and landmark piloting group were $5.31,5.17$, and 4.43 , respectively. A Tukey HSD post hoc test showed that none of these groups differed significantly from another. During the second stage of learning (Trial Blocks 4-6), the mean arm entries were
6.53 for the control group, 1.42 for the beacon homing group, and 3.33 for the landmark piloting group. The mean for the beacon homing group was significantly lower than the means for the control and landmark piloting groups $[q(30) \geq 6.40]$. The latter two groups' means were not significantly different from each other. In the third stage of learning (Trial Blocks 7-10), mean arm entries were 4.94, 1.23, and 3.77 for the control, beacon homing, and landmark piloting groups, respectively; the beacon homing group mean was significantly lower than the control and landmark piloting groups means $[q(30) \geq$ $8.46]$, but the control and landmark piloting groups' means did not differ significantly.

Over the three successive trial blocks, the frequency of entrances into nonreward arms was determined for rats in the landmark piloting group. These frequencies are shown in Figure 2 as percentages of total entries relative to the position of the landmark and goal $(\mathrm{G})$ on any given trial. There are two interesting things to note about these data. First, entries into nonreward black arms were not particularly lower or higher for arms near the landmark and reward arms than for the other black nonreward arms. Second, the white landmark arm was entered substantially less often than the black nonreward arms. The tendency to avoid the white arm was found also in the high number of arm entries shown by the beacon homing group on the first block of trials and by the control group's increase in arm entries on the fifth block of trials; the reward happened to be on the white arm during the fifth block.

To determine whether the rats in each group had used the landmark to find the reward arm, it was necessary to

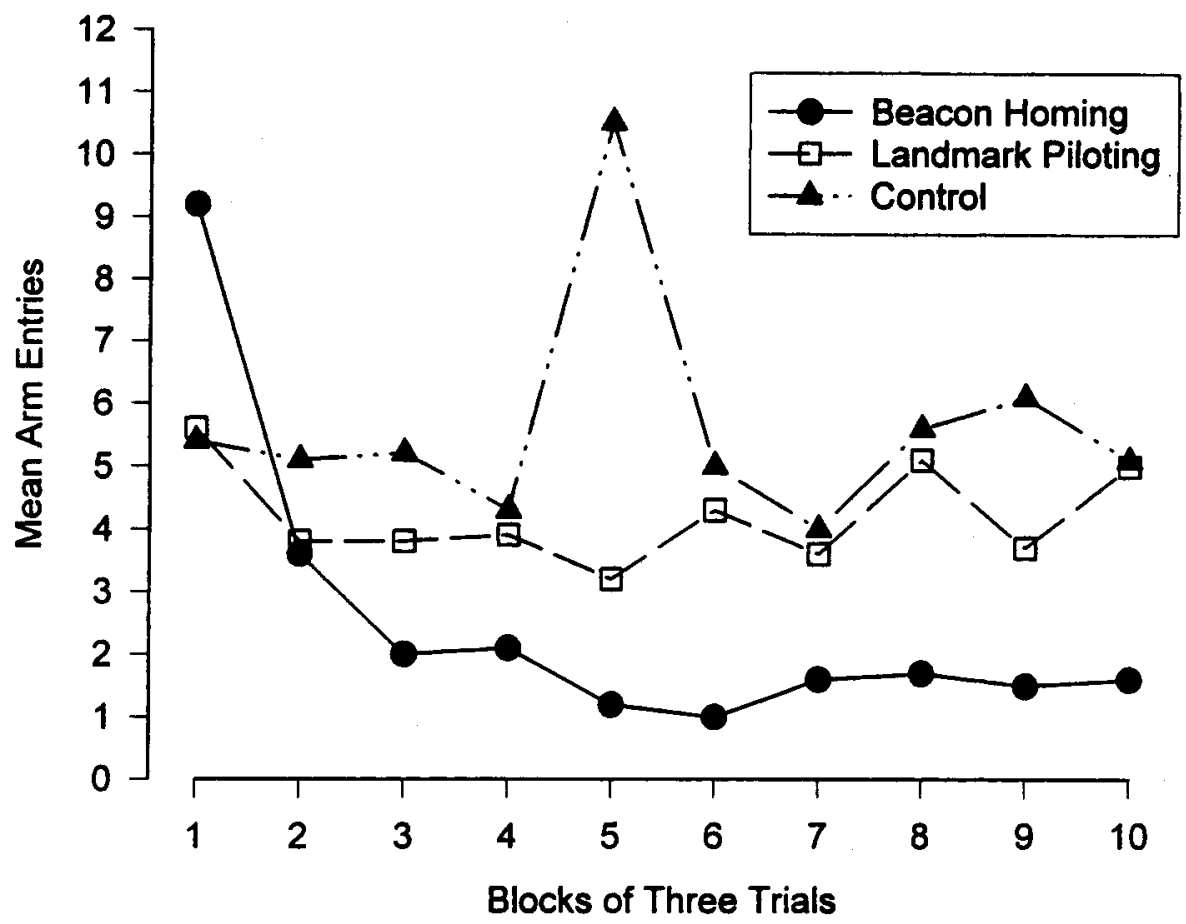

Figure 1. Mean arm entries required by three groups to locate the goal arm with a single intramaze beacon or landmark in Experiment 1. 
Trial Blocks 1 - 3

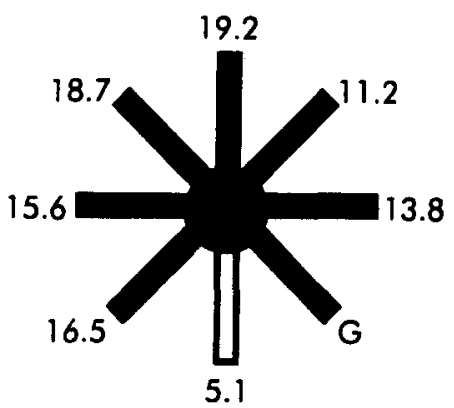

Trial Blocks 4 - 6

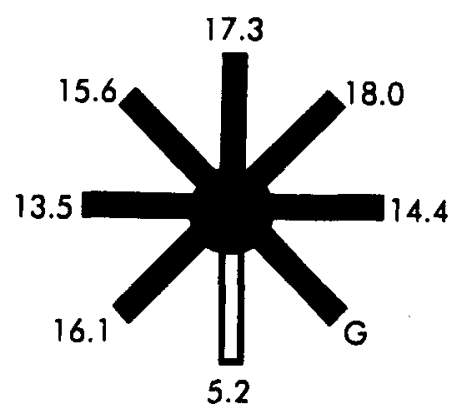

Trial Blocks 7 - 10

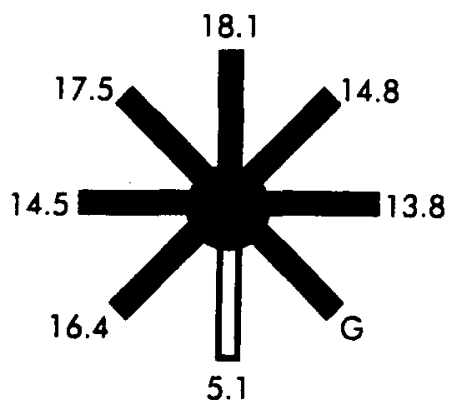

Figure 2. The distribution of error choices between maze arms made by the landmark piloting group in Experiment 1. The percentage choice of each arm is shown relative to the positions of the landmark and goal arms.

calculate how many arm entries could be expected to result in the reward's being found by chance. If each arm on a radial maze has an equal chance of entry, this expected value would be $(n+1) / 2$, where $n$ is the number of arms on the maze. Therefore, on an eight-arm maze, the number of arm entries expected by chance to result in the finding of the reward arm would be 4.5. The mean of 1.23 arm entries made by the beacon homing group was found to be significantly lower $[t(3)=42.17]$ than 4.5 .

In the case of the control and landmark piloting groups, the tendency to enter a white arm was lower than the tendency to enter the black nonreward arms, and therefore the value of $4.5 \mathrm{arm}$ entries was an overestimation of the chance value. A Monte Carlo program was designed to determine an appropriate chance level against which to test the control and landmark piloting groups' mean arm entries. Since the goal arm was not included in the calculations of percentages of nonreward arm choices, it was assigned the mean of the percentages of entries into the six nonreward black arms for Trial Blocks 7-10 (see Figure 2). The percentages of entries into each of the eight arms were then rescaled to sum to $100 \%$, and a simulation of 1,000 test trials was run, based on these percent- ages of entrance into each arm. Each simulation involv random selection of arms weighted by the observed $p$ centages of choice until the goal arm was chosen. W the initial percentage of entries to the landmark arm at $5.1 \%$, the number of arm entries for which rats col be expected to enter the reward arm by chance was 4 The observed mean number of arm entries over $\mathrm{Tr}$ Blocks 7-10 was 4.94 for the control group and 3.771 the landmark piloting group. These values did not dif significantly from the chance value of 4.1. This result $i$ dicated that the landmark piloting group was not able use the landmark to find reward faster than would be $e$ pected by chance alone.

\section{Discussion}

The results of this experiment show that the rats quick learned to use the white arm as a beacon to track rewal Although the rats in the beacon homing group initial showed an aversion to entering the white arm, within few trial blocks they chose this arm first on almost eve trial. Of considerable interest is the observation that $t$ ] rats in the landmark piloting group did not learn to $u$ the relationship between the white landmark arm and $\mathrm{t}]$ 
reward arm to track the reward. In fact, the data in Figure 2 suggest that these rats did not even tend to make more incorrect entries to arms near the landmark than to other arms on the maze. We designed the remaining experiments reported here to find out whether conditions could be arranged so that rats would learn the relationship between a landmark and a goal on the radial maze.

\section{EXPERIMENT 2}

The landmark used in Experiment 1 was an intramaze cue that consisted of a white arm that extended from the point at which a rat made its decision to enter an arm to the location of the food cup. In Experiment 2, we explored the possibility that a more localized extramaze cue would be more effective as a landmark by which rats could pilot their way to a goal location. The same experimental design was used as in Experiment 1, except that the landmark was a white disk positioned at the end of one arm of the maze.

\section{Method}

Subjects. Thirteen experimentally naive male Long-Evans hooded rats were tested. They were maintained under the same conditions as in Experiment 1.

Apparatus. The radial maze and testing room were the same ones that were used in Experiment 1. The landmark was a white circular plastic disk, $15 \mathrm{~cm}$ in diameter, mounted on the end of a piece of wooden doweling. The doweling had a wooden base and could be moved easily from arm to arm. When placed in position for a trial, the white disk sat $3 \mathrm{~cm}$ from the end of an arm and extended $1 \mathrm{~cm}$ below and $14 \mathrm{~cm}$ above the level of the arm.
Procedure. The general procedure was identical to that in Experiment 1 . Following an initial 2 -week pretraining period, the rats were assigned randomly to three groups. The beacon homing group contained 5 rats, the landmark piloting group contained 4 rats, and the control group contained 4 rats. The white disk was moved randomly from arm to arm between trials according to different sequences for each rat. The food reward of a chocolate chip was always placed in the food cup on the arm with the white disk for the beacon homing group and in the food cup on the arm directly to the left of the arm with the white disk for the landmark piloting group. For the control group, the locations of the white disk and the reward were independently determined and thus were unrelated.

The rats were tested for 2 trials per day, with a $10-\mathrm{min}$ intertrial interval. After $3^{5}$ trials of testing, concerns arose about the perceptual saliency of the white disk, since it was seen against the tan walls of the testing room. To ensure maximum contrast between the landmark and its background, black bristol board was affixed to the walls at the height of the maze. Testing under this condition was carried out over Trials $40-57$

\section{Results}

Mean arm entries for rats to find the reward arm are plotted as a function of 19 three-trial blocks in Figure 3. A groups $\times$ blocks of trials ANOVA revealed significant effects of groups $[F(2,10)=12.60]$ and blocks of trials $[F(2,20)=8.44]$, but a nonsignificant groups $\times$ blocks of trials interaction $[F(4,20)=2.23]$.

Performance across trials was divided into three stages, Trial Blocks 1-6, 7-13, and 14-19 (the final period of testing when the black bristol board had been affixed to the test room walls). During Trial Blocks 1-6, the beacon homing group required significantly fewer arm entries to find reward than did the control group and the land-

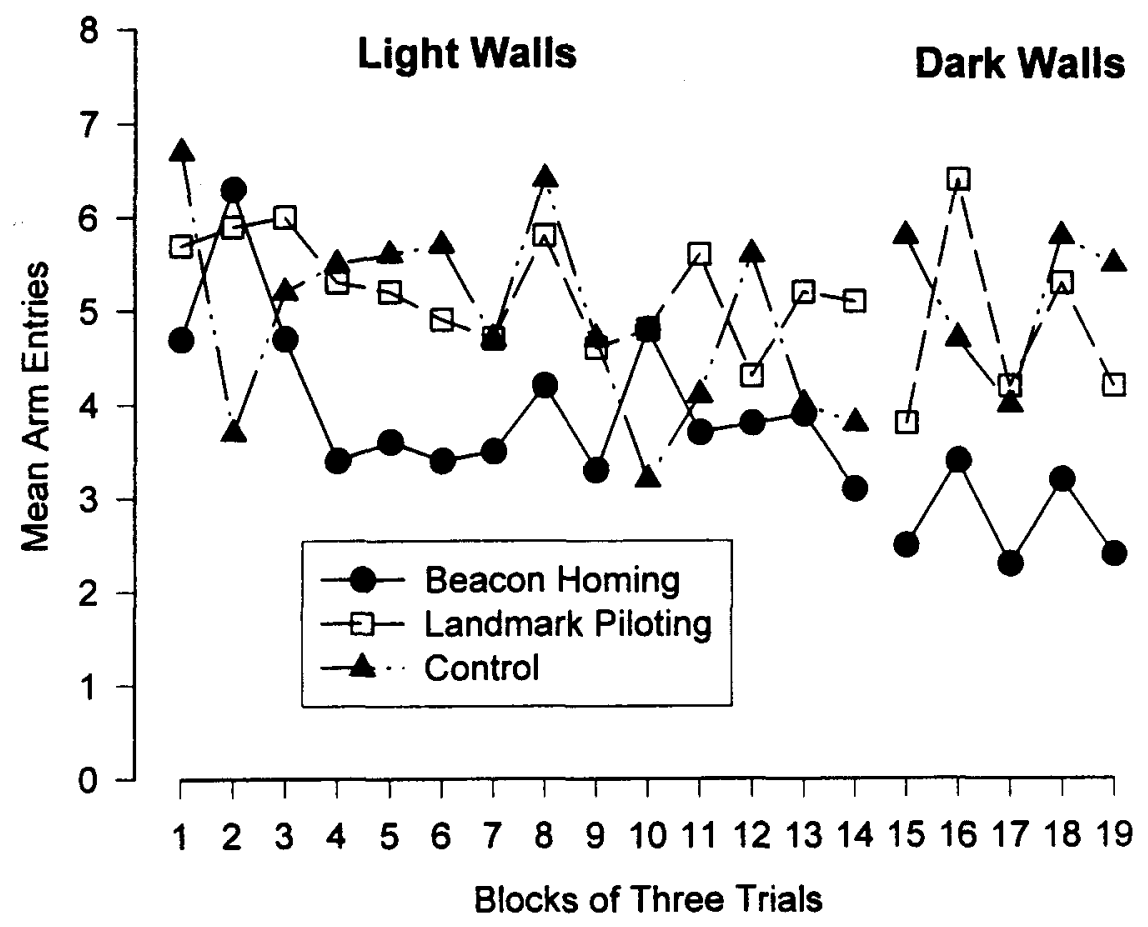

Figure 3. Mean arm entries required by three groups to locate the goal arm with a single extramaze beacon or landmark in Experiment 2. 
mark piloting group $[q(30) \geq 7.14]$, but the control and landmark piloting groups did not differ significantly from each other. The same pattern of differences was seen in Trial Blocks 7-13, in which the beacon homing group made significantly fewer mean arm entries than both the control and landmark piloting groups $[q(30) \geq$ $6.65]$, and the control and landmark piloting groups did not differ significantly from each other. These effects appeared again in Trial Blocks 14-19, with the beacon homing group significantly lower than the control and landmark piloting groups $[q(30) \geq 3.33]$, and no significant difference was found between the control and landmark piloting groups.

Figure 4 shows the distribution of arm entries made prior to entering the reward arm for rats in the landmark piloting group. During Trial Blocks $1-13$ and Trial Blocks 14-19, there was no tendency to avoid the landmark arm, as there had been in Experiment 1. The number of arm entries to find reward by chance was then set at 4.5 entries. Over Trial Blocks 1-6, 7-13, and 14-19, the means of arm entries to reward for the control group were 5.46, 4.61 , and 4.94 , respectively, and the means of arm entries

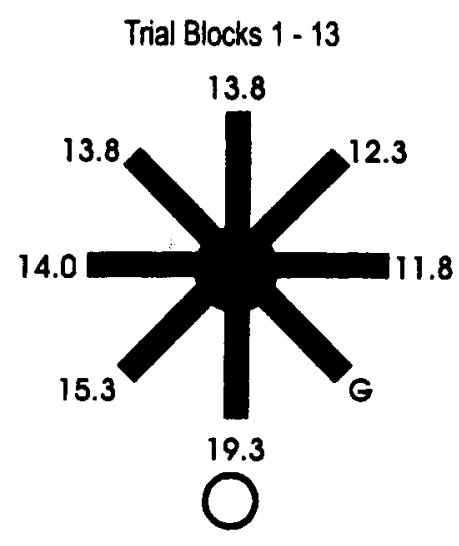

Trial Blocks $14-19$

(Blk Background)

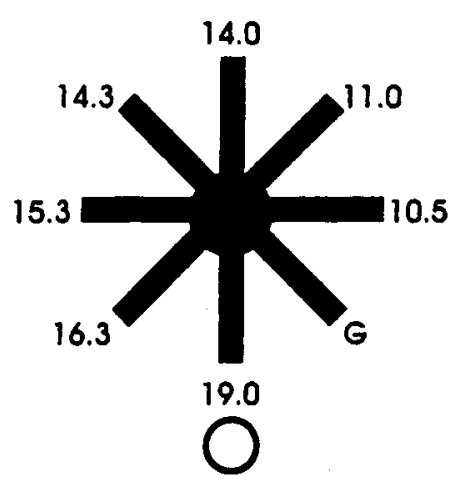

Figure 4. The distribution of error choices between maze arms made by the landmark piloting group in Experiment 2. The percentage choice of each arm is shown relative to the positions of the landmark and goal arms. to reward for the landmark piloting group were $5.39,4.98$ and 4.98 , respectively. None of these means differed sig nificantly from the chance level of 4.5 arm entries; it $i$ clear that neither the control nor the landmark pilotin groups found the reward arm below chance expectancy

Although the group comparisons suggested that th beacon homing group had learned the task better than th control and landmark piloting groups, $t$ tests showed tha the beacon homing group's mean arm entries durin Trial Blocks $1-6(M=4.22)$ and $7-13(M=3.78)$ wer not significantly lower than the chance level of $4.5[t$. 1.0]. Once a black background was put behind the whit landmark in Trial Blocks 14-19, the beacon homin group's performance dropped to a mean of $2.63 \mathrm{arm}$ en tries to find reward; this value was significantly belor $4.5 \mathrm{arm}$ entries $[t(4)=7.87]$.

\section{Discussion}

Although the rats in the beacon homing group did no find the reward as quickly in this experiment as the bea con homing rats in Experiment 1, the overall patterns 0 results from the two experiments are highly similar. Th beacon homing groups learned to approach the beaco: for reward, but the landmark piloting groups showed $n$ ability to use the relationship between the landmark an goal to find the reward sooner than expected by chanc alone. The landmark piloting rats did no better with al extramaze landmark than with an intramaze landmark.

Two observations drawn from Figure 4 are of interes 1 As in Experiment 1, there does not appear to have bee 1 a tendency in the landmark piloting group to cluster arn visits near the landmark arm, as one might have expecter if the animals had viewed the landmark as a salient cu for reward at a nearby location. Second, in contrast to th results shown in Figure 2 for the landmark piloting grou] in Experiment 1, the rats did not avoid the landmark arm if anything, they showed a slight preference for it. This ob servation suggests that the rats did not associate the land mark arm with the absence of reward in either Experiment or 2. The rats' avoidance of the white arm in Experiment was probably caused by an aversion to the white arm.

\section{EXPERIMENT 3}

It may have been particularly difficult for the rats in thi landmark piloting groups in Experiments 1 and 2 to learı the relationship between the landmark and goal becausi the locations of the landmark were moved continuall: between trials from the beginning of the experiment. I animals are biased to disregard the movement of land marks, as Biegler and Morris (1993) suggest, the fact tha the landmark was moved from the outset of testing ma: have led the rats to rapidly discount its importance. Ii Experiment 3, we explored the possibility that the rat would learn the relationship between the landmark anc goal if the landmark stayed in a fixed position during at initial period of training. If the rats attended to a fixer landmark and learned its relationship to reward, they migh then use it as a cue when it was moved about in space. 
Three groups of rats were tested in Experiment 3. For Group 1, the control group, a reward was placed on the same maze arm for 34 trials; the position of a white arm cover landmark was changed randomly from arm to arm between trials. For each rat in Group 2, the reward was placed on the same arm of the maze for 62 trials, with the landmark on the arm immediately to the right of the goal. Over the next 30 trials of testing, the landmark and goal were moved randomly from one arm to another between trials. The conditions of Group 2 allowed us to test the hypothesis that a period of training with a stable landmark and goal would facilitate goal tracking when the landmark and goal changed locations. The rats in Group 3 underwent similar training to that for those in Group 2, except that these rats were given two types of tests that were followed by retraining during the initial 62 trials in which the reward was always at a fixed location. These tests consisted of examining the rats' behavior when the landmark was either removed from the maze or placed on the arm opposite to its training position.

\section{Method}

Subjects. Sixteen experimentally naive male Long-Evans hooded rats served as subjects.

They were maintained in the same manner as in Experiments 1 and 2.

Apparatus. The same black eight-arm radial maze that was used in the first two experiments was used in Experiment 3, but it was placed in a different testing room, which measured $5 \times 5 \mathrm{~m}$. Numerous extramaze cues were available, including a second black radial maze, a table, a sink, a chair, a door, and an audio speaker. The intramaze landmark was the same white metal arm that served as the intramaze landmark in Experiment 1.

Procedure. The subjects were assigned randomly to three groups, Group $1(n=5)$, Group $2(n=6)$, and Group $3(n=5)$. For all groups, and throughout all phases of the experiment, only one arm of the maze was baited with a chocolate chip on each trial. Each rat in all three groups was assigned a different goal arm on which reward was placed on every trial during initial training.

For the rats in Group 1, the same arm contained reward throughout training. The white landmark had no predictive relationship to the location of reward, because it was moved randomly between arms over 34 trials of training. At this point, the testing of Group 1 rats was discontinued.

For the rats in Group 2, the white landmark was placed on the same arm, immediately to the right of the reward arm, throughout the initial 62 trials of training. For the remaining trials (Trials 63-92), the landmark and goal were moved randomly between trials, with the reward always on the arm directly to the left of the arm containing the landmark.

The rats in Group 3 were trained as were the rats in Group 2, with the goal and landmark in fixed locations, for Trials 1-26. Over Trials $27-34$, the landmark was removed from the maze, and the rats continued to have the reward placed on the goal arm. Retraining was done over Trials 35-42, with the landmark and goal placed on the same arms as in the initial training trials. Over Trials 43-48, the landmark was placed on the arm directly opposite to its training position on the maze. Another retraining period was carried out over Trials 49-62, with the landmark and goal in the original training positions. For Trials 63-92, the rats in Group 3 were tested with the same procedure as that for the rats in Group 2. The landmark and goal were moved randomly between trials, with the reward always placed to the immediate left of the landmark. The rats were trained with two trials per day and a 15 -min interval between trials.

\section{Results}

The mean number of arm entries required for rats to find the reward is shown as a function of two-trial blocks in Figure 5. The curves suggest that all three groups improved over Trials 1-26. A groups $\times$ blocks of trials ANOVA was performed on arm entries and revealed a significant effect of blocks of trials $[F(6,70)=12.25]$, but a nonsignificant effect of groups $(F<1.0)$. A significant groups $\times$ blocks of trials interaction was found $[F(11,70)=2.12]$ and appeared to arise from the erratic nature of Group l's curve during the initial stages of training. Over Trials $23-26$, the mean arm entries required for rats to enter the goal arm were $1.78,1.92$, and 1.86 for Groups 1, 2, and 3, respectively. Since the rats in all three groups showed a tendency to avoid the white landmark arm, as the rats did in Experiment 1, the number of entries required for rats to enter the goal arm by chance was set at the value of $4.1 \mathrm{arm}$ entries as in Experiment 1. The mean over Trials 23-26 was significantly below 4.1 for all three groups $[t(4) \geq 6.54]$.

When the landmark was removed from the maze on Trials 27-34 in Group 3, there was no disruption in rewardfinding accuracy, as can be seen in Figure 5. The mean arm entries required for rats to find reward with the landmark gone was 1.90 , and this value did not differ significantly from the value of 1.86 required on Trials $23-26$ $[t(4)=1.08]$. Similarly, placing the landmark on the opposite side of the maze during Trials $43-48$ had little effect on performance. The mean arm entries required for rats to find reward with the landmark on the opposite side of the maze was 2.07 ; this mean did not differ significantly from the mean of 1.67 arm entries that were required during the retraining period on Trials $35-42[t(4)=1.08]$. The means for both the landmark-removal and landmarkopposite tests were significantly lower than chance expectancy levels $[t(4) \geq 6.95]$.

During Trials 63-92, in which rats had to track a moving landmark and goal, mean arm entries that were needed for rats to find the goal arm increased substantially for both Group 2 and Group 3. Group 2 required 2.06 mean arm entries to find the reward during Trials 49-62; the mean arm entries on Trials 63-92 increased significantly to $4.78[t(5)=5.82]$. Similarly, Group 3 's mean arm entries increased significantly from 1.96 during the retraining period to 4.13 over Trials $63-92[t(4)=4.08]$. The means of both groups during the moving-landmark phase did not differ significantly from the value expected by chance.

\section{Discussion}

All groups clearly learned the location of the reward when it was consistently placed on the same arm. The results of the landmark-removal and landmark-opposite tests that were given to Group 3 were instructive. In neither 


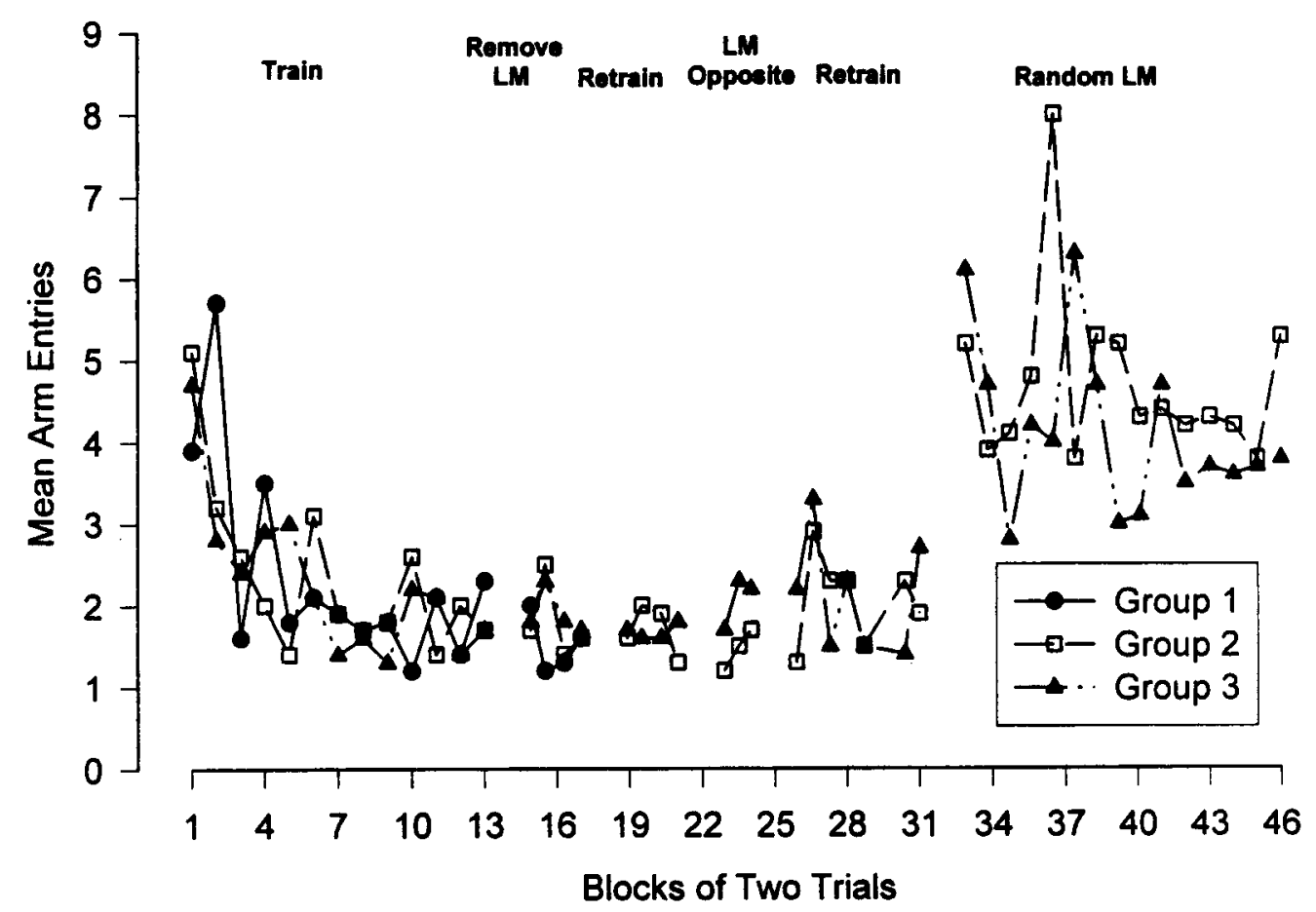

Figure 5. Mean arm entries required by three groups to locate the goal arm at successive stages of training in Experiment 3.

case did the landmark change produce a significant decrease in performance. This finding suggests that the rats used extramaze cues to pinpoint the location of reward. Either the rats did not attend to the relationship between the intramaze landmark and the goal, or, if they did, their learning was overshadowed by control of the extramaze cues (Diez-Chamizo, Sterio, \& Mackintosh, 1985).

The hypothesis that rats would learn to track the location of the randomly moving goal if first given a prolonged training period with the landmark and goal in fixed positions was not supported. The failure of Groups 2 and 3 to track the goal arm above chance expectancy during Trials 63-92 was not surprising, given Group 3's failure to show any effect of landmark removal or landmark shifting on the earlier tests. All three of the experiments thus far performed failed to yield any evidence that rats could learn to track a moving goal arm on the radial maze that had a constant spatial relationship to a landmark.

\section{EXPERIMENT 4}

Two concerns about the preceding experiments were addressed in Experiment 4. One concern was that the rats' failure to track the goal on the basis of its relationship to a landmark in Experiments 1-3 was the consequence of using only a single landmark. Because research with rats (Biegler \& Morris, 1996; Greene \& Cook, 1997; Rodrigo, Chamizo, McLaren, \& Mackintosh, 1997), gerbils (Collett, Cartwright, \& Smith, 1986), hamsters (Poucet, Chapuis, Durup, \& Thinus-Blanc, 1986), and Clark's nutcrackers (Kamil \& Jones, 1997) suggests that animal remember locations relative to multiple landmarks, it i possible that the rats needed to perceive the location of reward arm on the radial maze relative to more than on landmark in order to track the position of the goal. Thre groups of rats were studied in Experiment 4, and eacl group was trained with two landmarks. One landmark wa the white arm cover that was used in Experiments 1 an 3 , and the other landmark was an arm cover painted wit] alternating black and white stripes. For two groups, th landmarks were placed in a V configuration, with the re ward arm always in the middle, between the two land mark arms. One group (stable-V group) was trained witl the goal and landmark at fixed locations for a number o trials before they were tested with landmarks that changer locations. Another group (random-V group) was traine from the beginning of the experiment with landmarks anc goal that were moved randomly from one set of arms t another between trials. A comparison between these tw groups should indicate whether training with static land marks would be beneficial to the acquisition of goa tracking. A third group was trained with the landmark separated by two black arms (2-over group), with rewarc always placed on the arm immediately to the right of th left landmark (as viewed from the center of the maze) This group was tested in order to determine whether th rats would track the goal better with landmarks closer to gether or farther apart.

The second concern addressed in Experiment 4 wa: the importance of extramaze cues. The relationship be 
tween the goal and the landmarks that were used in Experiments 1-3 might not have been learned because the relationship between the goal and extramaze room cues overshadowed the experimenter's landmark. If this was the case, then reducing access to these room cues should increase the saliency of the landmarks manipulated by the experimenter. In Experiment 4, a dark curtain surrounded the radial maze and thus screened out most of the extramaze room cues.

\section{Method}

Subjects. The subjects were 15 experimentally naive Long-Evans hooded rats that were maintained under the same conditions as in the first three experiments.

Apparatus. The rats were pretrained on the same black, eightarm radial maze that was used in Experiment 3 and in the same room as in Experiment 3 . The maze was then moved to a new room $(4.5 \times 4.0 \mathrm{~m})$ for formal testing. The maze was surrounded by a dark green curtain that hung from a circular track on the ceiling ( $195 \mathrm{~cm}$ in diameter) to a height of $20 \mathrm{~cm}$ below the ends of the maze arms. Three entrances (north, south, and west) through the curtain into the maze area were available.

Two intramaze landmarks were used. Landmark 1 was the white arm cover used in Experiments 1 and 3. Landmark 2 was an arm cover that was made visually distinctive from Landmark 1 by painting alternating black and white stripes ( $1.5 \mathrm{~cm}$ wide) across its width.

Procedure. The 15 rats were assigned randomly to three groups, with 5 rats in each group. As in the previous experiments, only one arm on the maze contained a chocolate chip reward on each trial. In the stable- $V$ group and the random $-V$ group, the reward was always located on the arm between the two landmark arms, with the white landmark always on the arm to left of the goal arm and the striped landmark always on the arm to the right of the goal arm. In the 2over group, the white landmark was always on the arm immediately to the left of the goal arm, and the striped landmark was always on the arm two arms over to the right of the goal arm.

Each rat in the stable- $V$ and 2-over groups was assigned a different goal arm, and the reward and landmarks were placed on the same arms for Trials $1-27$. On trials $28-33$, the landmarks were removed from the maze for both of these groups, but reward was still found on the goal arm. During Trials 34-39, both groups were retrained with the landmarks and goal in the same fixed positions that were used over trials $\mathbf{l}-27$. In the final stage of the experiment (Trials 40-57), the landmarks -goal array was moved randomly between arms in both the stable- $V$ and 2-over groups.

For the rats in the random- $V$ group, the position of the landmarks and goal moved randomly between arms over the entire 57 trials of the experiment.

The general testing procedure was the same for all groups throughout all phases of the experiment. On each trial, the experimenter carried a rat in a black plastic tube into the testing room and then entered the curtained area; entrance into the curtained area varied randomly over trials between the north, south, and west entrances. As a further control for the use of vestibular cues, the rat was given four $360^{\circ}$ rotations in the carrying tube before it was placed on the center of the maze. The rat was then allowed to traverse the maze freely until it located the goal arm. The rats were tested in this manner on one trial per day.

\section{Results}

The mean arm entries for rats to find the goal are plotted as a function of blocks of three trials in Figure 6, with separate sets of curves for the locate goal, remove land-

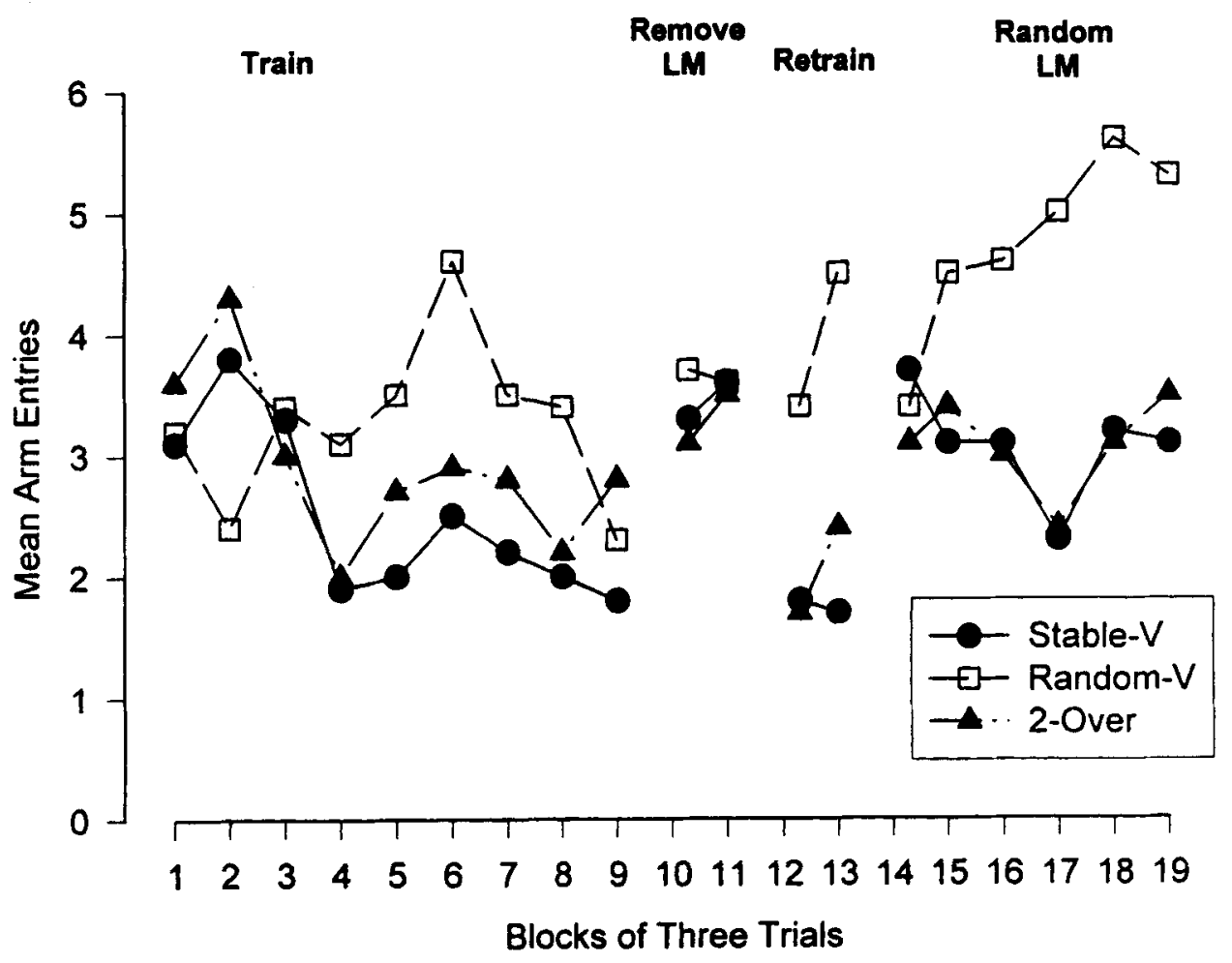

Figure 6. Mean arm entries required by three groups to locate the goal arm at successive stages of training in Experiment 4. 
mark, retrain, and random landmark phases of the experiment. Throughout these phases of the experiment, it was found that the rats in each of the groups avoided entries into the landmark arms to some degree. The distributions of entries into landmark and nonlandmark arms were determined for each group at each phase of the experiment. Monte Carlo simulations were then run on each of these distributions to find the number of reward arm entries expected by chance. The expected values are shown for each condition at each phase of the experiment in Table 1.

A groups $\times$ blocks of trials ANOVA was performed on arm entries needed for rats to find the goal over Trials 1-27. The analysis yielded significant effects of blocks of trials $[F(4,47)=2.98]$ and groups $[F(2,12)=$ 5.67], but a nonsignificant groups $\times$ blocks of trials interaction $[F(8,47)=1.89]$. Over Trials 19-27, the mean arm entries required by the stable- $\mathrm{V}, 2$-over, and random$\mathrm{V}$ groups were $1.90,2.50$, and 3.10 , respectively. The rats in both the stable-V and 2-over groups found the reward in significantly fewer mean arm entries than expected by chance $[t(4) \geq 7.69]$ (see Table 1 ), but the mean entries required by the rats in the random- $V$ group were not significantly lower than the level of chance expectancy.

When the landmarks were removed from the maze on Trials 28-33, the stable- $V$ group's mean arm entries increased significantly from 1.90 to $3.63[t(4)=10.04]$. Although the 2-over group's mean arm entries also increased from 2.50 to 3.50 , this increase was not significant $[t(4)=1.27]$. The distribution of arm entries for both the stable- $V$ and 2-over groups showed that, in the absence of the landmarks, the rats chose equally among the eight arms; thus, a chance level of 4.5 arm entries was used for both groups during this phase of the experiment. The mean of 3.63 for the stable- $\mathrm{V}$ group was significantly lower than $4.5[t(4)=2.88]$, but the mean of 3.50 for the 2-over group was not significantly different from 4.5. The random- $V$ group's landmarks were not removed from the maze, and the mean arm entries over Trials 28-33 $(M=3.67)$ did not differ significantly from the chance level of 3.6 arm entries.

Over Trials 34-39, the stable-V and 2-over groups underwent a retraining period with the landmarks returned to their original positions. Over these trials, the mean arm entries dropped to 1.76 for the stable- $V$ group and to 2.06 for the 2-over group. Both of these means were significantly lower than chance expectancy $[t(4) \geq 11.86]$. Dur-

Table 1

Mean Arm Entries Expected by Random Selection of Arms Based on Monte Carlo Simulation Runs for Each Group at Each Stage of Training in Experiment 4

\begin{tabular}{lccc} 
& \multicolumn{3}{c}{ Group } \\
\cline { 2 - 4 } Stage of Training & Stable-V & Random-V & 2-Over \\
\hline Locate goal & 3.75 & 3.60 & 3.50 \\
Remove landmark & 4.50 & 3.60 & 4.50 \\
Retrain & 3.74 & 3.86 & 3.50 \\
Random landmark & 3.95 & 4.39 & 3.50 \\
\hline
\end{tabular}

ing this period, the random- $V$ group made 3.84 mear arm entries, a value that did not differ significantly from the chance level of 3.86 .

When the landmark-goal arrays were moved randomly about the maze during Trials $40-57$, the stable- $V$ group's mean arm entries increased to 3.17 , but this mean was significantly lower than the chance level ot $3.95[t(4)=6.06]$. The 2-over group's mean arm entries increased to 3.12 , but this mean did not differ significantly from the chance level of 3.50. Finally, the rats ir the random- $V$ group took 4.72 mean arm entries to finc reward, but this mean did not differ significantly from the chance level of 4.39. Therefore, only the stable- $V$ group showed evidence of being able to track the goal location at a level below chance expectancy.

\section{Discussion}

The purpose of this experiment was to determine whether rats could learn a relationship between moving landmarks and a goal when two landmarks were used. The stable- $V$ group did yield evidence of significant goal tracking. It appears that two landmarks in a $\mathrm{V}$ configuration on each side of the goal arm were required and that the rats had to have initial training with the landmarks and goal in a fixed location over a number of trials for tracking to appear. When tracking did appear, however, it was not impressive. The rats in the stable- $V$ group still took over three arm entries to find the reward arm. When compared with performance of the beacon group in Experiment 1 , this level of accuracy suggests that it was much easier for the rats to use a single beacon to find the goal than to use the relationship between two landmarks and the reward arm to find the goal.

\section{EXPERIMENT 5}

Although the stable- $\mathrm{V}$ group in Experiment 4 did find the reward arm significantly sooner than chance expectancy, the number of arms entered was greater than three and was less than one arm entry below the chance level. We conducted Experiment 5 in an attempt to find conditions under which better landmark piloting would occur in the radial maze.

Two groups of rats were trained under different conditions. For the half-maze group, initial maze choices were restricted to half the maze, or three arms. Three arms of the maze were blocked. Among the remaining five arms, two landmarks were placed in a $\mathrm{V}$ configuration on either side of the black reward arm. Thus, three arms among the five could serve as reward arms. It was anticipated that the rats might learn the goal's relationship to the landmarks better with a restricted set of choices. Eventually, these rats were tested for goal tracking on the full eight-arm maze.

In the experiments of A. D. L. Roberts and Pearce (1998), two procedures helped rats learn to track the relationship between a landmark and the goal platform in a swimming pool. First, the rats were given multiple trials on 
each session, with the landmark and goal in a constant location within the session but changed between sessions. Second, rats were given extinction trials on which both the landmark and goal were absent from the swimming tank. The extinction trials may have enhanced the rats' attention to the landmark. The second group of rats in our Experiment 5 , the full-maze group, was trained under similar conditions. The entire eight-arm maze was used, and the reward was always placed on an arm between two landmark arms that formed a V configuration. On each daily session, the rats received five trials. On three of the trials, the landmarks and goal were placed on the same arms. Between sessions, the positions of the landmarks and goal were changed. On the remaining two trials within each session, extinction training, in which the maze contained no landmarks and no reward, was given. It was anticipated that these conditions would be conducive to learning the landmarks-goal relationship on the radial maze, as they were in A. D. L. Roberts and Pearce's swimming tank.

\section{Method}

Subjects. Thirteen experimentally naive male Long-Evans hooded rats were used. The rats were maintained under the same conditions as in the first four experiments.

Apparatus. The black eight-arm radial maze used in Experiment 4 was used in Experiment 5. Training took place in an experimental room that measured $4.5 \times 4.5 \mathrm{~m}$. The radial maze was surrounded by a dark green curtain that hung from the ceiling to the floor. The same landmarks used in Experiment 4 were used in Experiment 5. Landmark 1 was the white arm cover, and Landmark 2 was the striped arm cover.
Procedure. The rats were divided randomly into two groups, the half-maze group $(n=7)$ and the full-maze group $(n=6)$. In both groups, only one arm on the maze was baited with one chocolate chip reward on each trial. The white landmark always was placed on the arm immediately to the left of the reward arm, and the striped landmark always was placed on the arm immediately to the right of the reward arm.

For the half-maze group, three of the arms on the maze were blocked off by wooden barriers placed at the arm entrances. Among the remaining five arms, any one of the three central arms could be the reward arm and have landmarks placed on each side of it. The reward arm was changed from trial to trial in a random order. Each rat was tested twice a day, with an intertrial interval of $5 \mathrm{~min}$. After 36 trials of training on the half maze, the rats were tested further on the entire eight-arm maze. The reward arm and landmarks were moved randomly between arms during the test period. The rats were tested for 9 days, 1 trial per day.

For the full-maze group, a different arm among the eight arms on the maze was chosen to contain reward on each daily session. On three of the five trials within a session, the chosen reward arm had the white landmark on the arm to its left and the striped landmark on the arm to its right. The other two trials within a session were extinction trials, in which no landmarks and no reward were placed on the maze. The first trial of a session was always a test trial with landmarks and goal. Two trials with landmarks and goal and two extinction trials then followed in random orders that varied from session to session. The full-maze group was tested for 21 daily sessions.

The same general testing procedure was used for both groups. On each trial, a rat was carried into the testing room in an enclosed wooden carrying cage. It was then rotated through $360^{\circ}$ four times in the carrying box. The experimenter then entered the maze area through the curtain surrounding the maze from one of three entrances (north, south, or west), removed the rat from the carrying box, and placed it on the center of the maze. For the half- and full-maze test

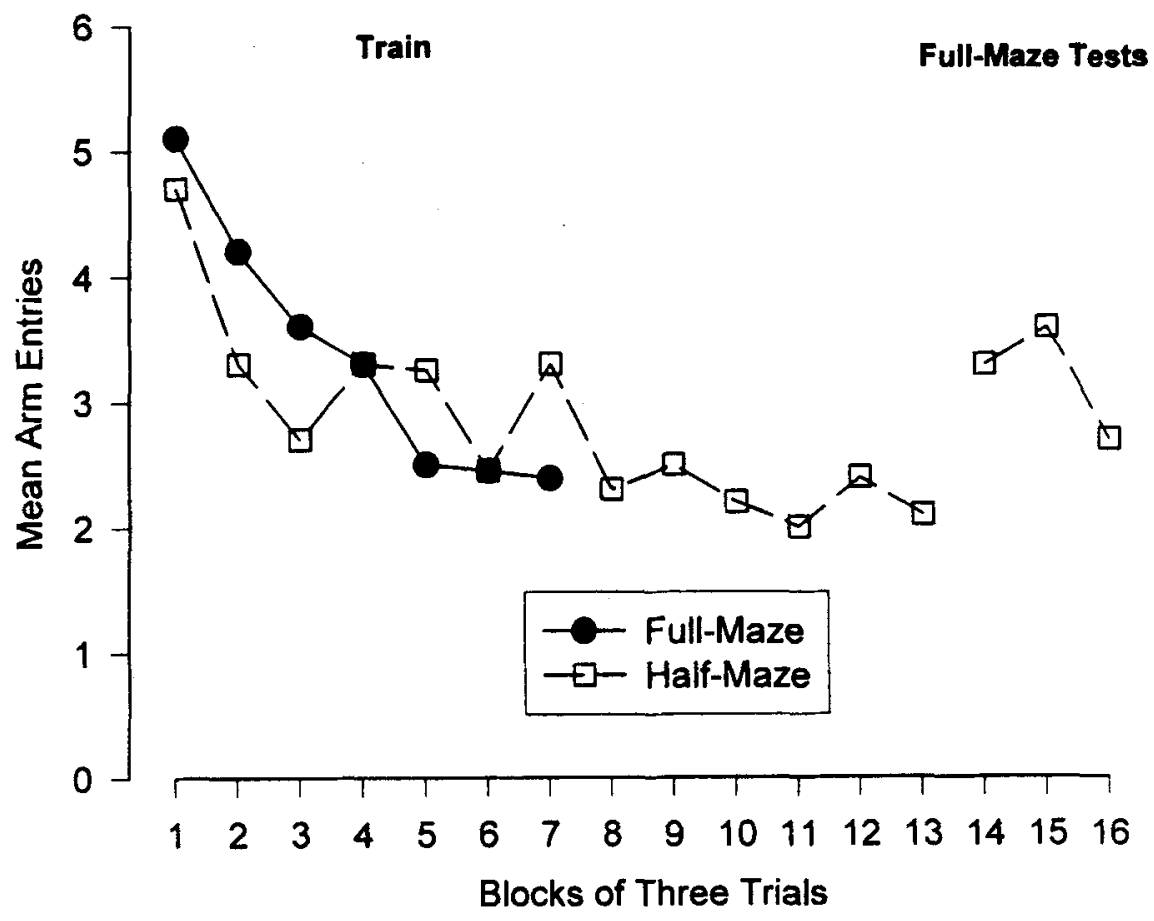

Figure 7. Mean arm entries required by two groups to locate the goal during training in Experiment 5. 
trials with the landmarks and goal, a rat was removed from the maze after it had entered the goal arm and consumed the reward. On extinction trials in the full-maze group, a rat was left on the maze until it had either entered all eight arms or been on the maze for $5 \mathrm{~min}$.

\section{Results}

Mean arm entries were averaged over three-trial blocks for both groups and are plotted in Figure 7. Only the arm entries on the first trial of a session were used to plot the curve for the full-maze group. For the first block of three trials, the half-maze group had a mean of 4.86 arm entries; by the last three-trial block on the half maze (Trials 34-36), mean arm entries had dropped to 2.0. The mean arm entries made by the full-maze group on the first block of three trials was 5.06, and, by the final three-trial block of testing (Trials 19-21), the mean number of arm entries was 2.39. One-way ANOVAs showed that the decrease in mean arm entries over trial blocks was significant in both the half-maze group $[F(12,72)=5.28]$ and the full-maze group $[F(6,30)=3.22]$.

To determine appropriate chance levels of arm entries required for rats to find the goal, distributions of error arm entries were observed for each group. The results showed that the rats in both groups did not exhibit the strong avoidance of the landmark arms that had been observed in the previous experiments. The rats in the halfmaze group made $22.49 \%$ of their entries into the white arm and $22.16 \%$ of their entries into the striped arm. These means are only slightly lower than the $27.19 \%$ and $28.16 \%$ of entries made into the two remaining black arms on the half maze. Because the rats did not avoid the landmark arms on the half maze, the chance level of arm entries to find reward was set at $(5+1) / 2=3$. The mean of $2.1 \mathrm{arm}$ entries made on the last block of three trials was significantly lower than the chance value $[t(6)=3.22]$.

In the full-maze group, the rats entered the white arm on $10.44 \%$ of error choices and entered the striped arm on $14.38 \%$ of error choices, as compared with a range of $13.78 \%-18.80 \%$ of error choices made into the remaining five black arms. Because the rats did not show avoidance of the landmark arms, the chance level of arm entries to find the goal was set at 4.5. The value of 2.39 mean arm entries made on the final block of three trials was significantly lower than the chance value of $4.5[t(5)=7.27]$.

The expanded-maze test trials given to the half-maze group with all eight arms available are shown as threetrial blocks on the right side of Figure 7. Mean arm entries increased from the half-maze phase, because the number of possible error arms had increased by three arms. The mean number of arm entries required for rats to find reward over Trials $37-45$ was 3.30 and was significantly lower than the chance value of 4.5 arm entries $[t(6)=6.12]$.

\section{Discussion}

The methods used in Experiment 5 were successful in producing better goal tracking than had been found in the previous four experiments. Although trained with a landmark-goal array that moved between trials from the beginning of the experiment, the rats in the half-m: group came to find the goal a whole arm entry earlier th chance expectancy. When switched to a full eight-a maze, the rats continued to track the moving goal at $m$ than an arm entry below chance expectancy.

The findings for the full-maze group were even $m$ successful. The combination of repeated tests with landmarks and goal in the same positions within a sess and intermixed extinction trials led the rats to accurat track a goal that changed position between sessions. the first test trial of each session, the rats improved tra ing to the point at which they were finding the goal $\mathrm{m}$ than two arm entries below chance expectancy; wh choosing among eight arms, the rats entered the goal a in slightly over two arm entries. These findings sugg that, given the appropriate training conditions, rats learn the relationship between moving landmarks an goal on the radial maze.

\section{EXPERIMENT 6}

The clear evidence that the rats learned to find a $\mathrm{g}$ placed between two landmarks in Experiment 5 raise further question. Does this effect occur only with the configuration of landmarks? It could be argued that black goal arm stands out perceptually as an isolated a between the white and striped landmarks and is theref approached as a beacon. If so, the effect seen in Expr ment 5 would be the same as that seen for the beac homing groups that were tested in Experiments 1 anc On the other hand, the conditions used in Experimer might reveal evidence of landmark piloting under la mark conditions that did not reveal accurate goal loc ization in Experiments 1-4.

Three groups of rats were used. One group was des nated the $\mathrm{V}$ group and was tested under the same con tions as was the full-maze group in Experiment 5. 1 results from the $\mathrm{V}$ group should indicate the replical ity of the goal-finding behavior that was seen in Exp iment 5. Because there was no evidence that the rats co find the goal sooner than chance expectation with landmark or with the 2-over pattern of two landmark: Experiments 1-4, one group was trained with a sin landmark and the other group was trained with landma separated by two black arms. These conditions should veal whether the rats would be capable of using th landmarks to find the goal when the training conditi adapted from A. D. L. Roberts and Pearce (1998) w used or whether rats could still fail to track the goal un these landmark conditions.

\section{Method}

Subjects. Twenty naive male Long-Evans hooded rats $x$ tested. They were maintained in the same manner as were the in Experiments 1-5.

Apparatus. The black eight-arm maze that was used in Exp ments 4 and 5 was also used in Experiment 6 . Testing was carried in the same room that was used in Experiment 5, with the maze 
rounded by a dark green curtain. The landmarks were the white and black-and-white striped arm covers that were used in previous experiments.

Procedure. Three groups of rats were formed, the 1-landmark group $(n=7)$, the $\mathrm{V}$ group $(n=6)$, and the 2-over group $(n=7)$. The reward used in this experiment was half of a piece of Frosted Cheerios breakfast cereal. The rats in each group were given five trials per day, with three reward trials and two extinction trials. The first trial of the day was always rewarded, and two of the remaining four trials, randomly chosen, were also reward trials. The remaining two trials were extinction trials, in which no landmarks and no reward were placed on the maze. The same arm of the maze contained the reward on each rewarded trial within a day, and the location of the reward arm was changed randomly between days.

For the 1-landmark group, the white arm cover was always placed on the arm immediately to the left of the reward arm. The white and striped arm covers were always placed on the arms immediately to the left and right of the reward arm on each trial for the $V$ group. In the 2-over group, the white cover was placed on the arm immediately to the left of the reward arm, and the striped cover was placed on the arm two arms to the right of the reward arm.

The three groups were tested for 30 days under the same conditions that were used in Experiment 5 . That is, the experimenter rotated the rat $360^{\circ}$ four times in the carrying box and then carried the rat to the maze through an entrance in the curtain. The entrance was changed randomly between trials.

\section{Results}

Figure 8 shows mean arm entries plotted as a function of blocks of three trials. Only arm entries on the initial trial of each day were used to plot these curves. The curves show improvement over the last four blocks of trials in the 1-landmark group and the V group, but not in the 2- over group. A groups $\times$ blocks of trials ANOVA revealed a significant drop in the number of arm entries over blocks of trials $[F(9,153)=4.13]$, but a nonsignificant effect of groups $[F(2,17)=1.77]$ and a nonsignificant groups $\times$ blocks of trials interaction $(F<1.00)$.

Calculation of the mean arm entries over Blocks 7-10 yielded means of 2.90, 2.90, and 4.24 for the 1-landmark, $\mathrm{V}$, and 2-over groups, respectively. These group means differed significantly $[F(2,17)=5.46]$, and comparisons between individual groups revealed that that number of entries for the 2-over group was significantly higher than those for the 1-landmark group $[t(12)=2.73]$ and the $V$ group $[t(11)=2.56]$. Because the rats in all three groups made errors frequently by entering landmark arms, 4.5 arm entries to obtain the reward was used as the chance level. The mean of $2.90 \mathrm{arm}$ entries was significantly lower than chance for both the 1-landmark group $[t(6)=6.05]$ and the $V$ group $[t(5)=7.54]$, but the mean of $4.24 \mathrm{arm}$ entries that was found in the 2-over group did not differ significantly from chance.

\section{Discussion}

The finding that the $\mathrm{V}$ group learned to find the goal arm significantly sooner than chance expectancy replicated the results of Experiment 5 and showed again that the rats used two landmarks to find food when the landmarks bracketed the goal arm. A further important finding was the discovery that the rats in the 1-landmark group also learned to find the reward arm below the chance level. Although the results of Experiments 1-3 suggested

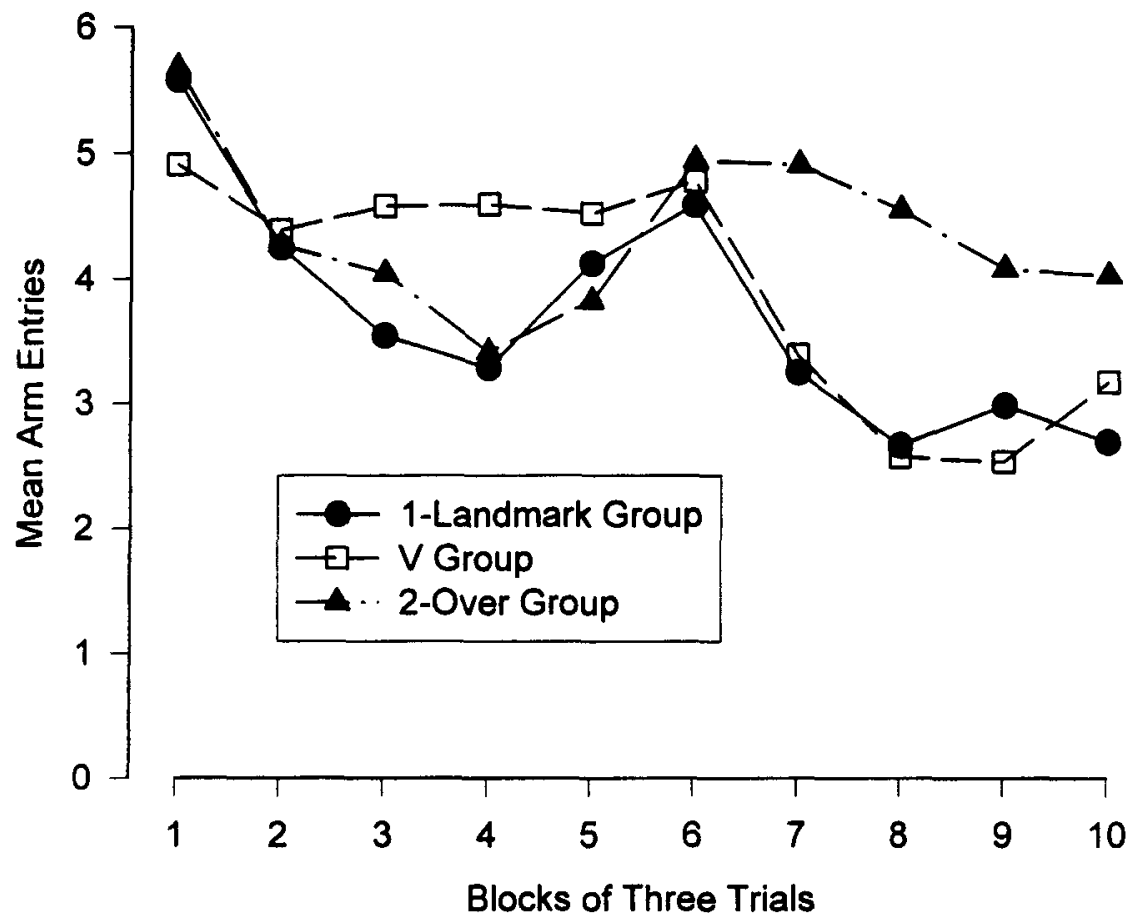

Figure 8. Mean arm entries required by three groups to locate the goal during training in Experiment 6. 
that the rats could not use the relationship between the goal arm and a single landmark adjacent to it to find food, this result indicated that those initial failures arose from testing conditions that were not optimal. When choice of the same arm was rewarded repeatedly within daily trials and extinction trials were intermixed with reward trials, an ability to use a single landmark was revealed.

The puzzling finding of Experiment 6 was the failure to find any evidence that the rats in the 2-over group learned to use the landmarks to find the goal arm. This finding agrees with the failure to find better than chance performance in the 2-over group that was tested in Experiment 4 . One possible explanation is that the relationships between the goal and landmark(s) were easier to learn in the 1-landmark and $V$ groups than in the 2-over group. In both the 1-landmark and V groups, the landmark(s) were adjacent to the goal arm; the rats could then encode the goal as immediately to the right of the white landmark in the 1-landmark group and as immediately to the right of the white landmark and/or immediately to the left of the striped landmark in the V group. In the 2-over group, however, the goal would have to have been coded as immediately to the right of the white landmark but more distant from the striped landmark. Perhaps this asymmetry of distances from the two landmarks made the coding task more difficult.

\section{GENERAL DISCUSSION}

We performed six experiments in order to examine the rats' ability to use landmarks as cues to food location on the radial maze. Although the radial maze has been used widely to study other problems in spatial cognition and memory in animals (W. A. Roberts, 1998a), questions about landmark piloting have been rarely addressed. The present experiments are particularly salient because the findings of other well-known spatial cognition paradigms have yielded inconsistent results. For example, Biegler and Morris (1993) found that rats could not use a moving landmark to locate food buried at a constant distance and direction from the landmark in an open field, but A. D. L. Roberts and Pearce (1998) discovered that rats were quite adept at using a moving landmark to find a platform hidden under the water in a swimming tank at a fixed distance and direction from the landmark. In the present experiments, we explored rats' use of distinctive cues that were placed on maze arms both as a beacon for food and as landmarks at a constant distance and direction from food that could be used to pilot toward the goal. The structure of the radial maze has two advantages for these types of study. First, because the rat must always make a choice of an arm while it is on the central platform, it cannot approach a landmark and then orient itself toward the goal from the landmark. The structure of the maze guarantees that the animal must use the relationship between the landmark and goal to make its choice. Second, choice from a central circular platforı ensures that the correct relationship between the lanc mark and the goal will always be seen by the subje without the use of a polarizing cue that is located somr where in the extramaze environment. In the single-lanc mark conditions, for example, reward was always on th arm to the left or to the right of the landmark, regardles of the compass direction in which the landmark arm we pointing.

The rats quickly learned to use a moving beacon $t$ find food on the radial maze. When a white cover we moved from arm to arm in Experiment 1, or a white dis was placed at the end of different arms in Experiment : the rats learned to enter the beacon arm early for food $\mathrm{r}$ ward. Just as striking was the inability of the rats in E? periments $1-3$ to use a single landmark as a cue to pils the location of reward. When reward was placed on th arm immediately to the left of a landmark that change positions between trials, the rats showed no evidence $c$ being able to find it sooner than expected by chance alon Even when two landmarks were introduced in Exper ment 4 , only slightly better than chance performance wc found for the group trained with a $V$ configuration $c$ landmarks.

In Experiments 5 and 6, conditions were found und which the rats showed clear evidence of piloting towar a goal by using landmarks. These conditions were adapte to the radial maze from the swimming tank experimen 1 of A. D. L. Roberts and Pearce (1998) and consisted c multiple trials within a session that kept the landmark(s and goal in the same locations. Extinction trials were ir terspersed among the rewarded trials, and the location of the landmark(s) and goal were changed randomly be tween daily sessions. The procedure of rotating an anims $360^{\circ}$ several times before each trial also was introduce in Experiments 5 and 6 and may have contributed to su perior performance by reducing the rats' reliance on ves tibular cues. Under these conditions, the rats learned $t$ find the goal in substantially fewer entries than the chanc level of arm entries. Furthermore, they did so both whe two landmarks were placed in a $V$ configuration aroun the reward arm (full-maze group in Experiment 5 an $\mathrm{V}$ group in Experiment 6) and when only one landmar was placed beside the goal arm (1-landmark group i Experiment 6). It may be that daily sessions with th landmark(s) and the goal in the same positions on re warded trials and with intermittent extinction trials ar particularly effective ways of making rats attend to th relationship between the landmarks and goal.

What are the theoretical implications of these find ings? For one, these findings, taken in conjunction wit] those of Biegler and Morris (1993, 1996), suggest tha rats may be more biologically prepared to use a singl moving beacon as a cue for food location as opposed $t$ a landmark that is placed at some fixed distance from : goal location. Although significant use of a single land 
mark to find the goal arm was found in Experiment 6, the rats still made 2.90 mean arm entries to find the food arm. In Experiment 1, the rats used a beacon to find food in only slightly more than a mean of one arm entry.

The findings of Experiments 1-5 seemed to be consistent with those of Biegler and Morris (1996) in that they suggest that piloting is easier for rats using two landmarks rather than one. When piloting with one and two landmarks was directly compared under optimal training conditions in Experiment 6, however, no difference in the final levels of accuracy was found between the 1-landmark group and the $V$ group that was trained with two landmarks. Thus, it appears that rats can pilot toward the reward arm on the radial maze as effectively with one landmark as with two. However, the findings with the 2-over groups in Experiments 4 and 6 suggest a caveat to this conclusion. Apparently, two landmarks are effective when they form a $V$ configuration that brackets the reward arm. When one of the landmarks is more distant from the reward arm than the other, they appear to lose their effectiveness.

How did the rats use landmarks to pilot toward the goal arm? When two landmarks formed a $\mathrm{V}$ around the goal arm, the rats could have learned an association between response and the configuration formed by the two landmarks (Sutherland \& Rudy, 1989). In this case, the configuration of a white landmark on the left and a striped landmark on the right would elicit a response of entering the arm that bisected this $\mathrm{V}$ pattern. This account might explain why the rats did not do better than chance with the 2-over configuration in Experiment 6; the goal arm in the 2-over group did not bisect the region between the two landmarks.

Other research suggests that animals that are faced with an array of landmarks that all have a fixed relationship to a goal may learn independent relationships with one or more of the items in the array (Collett et al., 1986; Spetch, Cheng, \& MacDonald, 1996; Spetch et al., 1997). Thus, the rats in the $\mathrm{V}$ group could have learned two separate landmark-goal relationships, one in which the goal was to the right of the white landmark and the other in which the goal was to the left of the striped landmark. The fact that both of these relationships involved adjacent arms might explain the relatively poor performance of the 2-over group, in which asymmetrical relationships would have to have been learned. In the case of a single landmark, however, only one of these relationships can be used. If the rats were using relationships between individual landmarks and the goal, it could be predicted that the summed effect of two separate associations would lead to better piloting than the effect of only one landmark. The equivalent performance found in the 1landmark group and the $\mathrm{V}$ group in Experiment 6 does not support this prediction.

Whether the rats responded to the configuration of landmarks or to independent landmark-goal relationships can only be determined by further research. For ex- ample, rats might be trained to track a goal between landmarks in the $\mathrm{V}$ pattern and then be given probe trials in which the landmarks were reversed or presented singly. The configurational theory suggests that performance should decrease to chance when the configuration is destroyed. If rats use independent landmark-goal associations, however, we would expect the rats to show some tendency to go to the arm to the right of the white landmark and to the arm to the left of the striped landmark.

\section{REFERENCES}

Biegler, R., \& Morris, R. G. M. (1993). Landmark stability is a prerequisite for spatial but not discrimination learning. Nature, 361 , 631-633.

Biegler, R., \& Morris, R. G. M. (1996). Landmark stability: Studies exploring whether the perceived stability of the environment influences spatial representation. Journal of Experimental Biology, 199, 187-193.

Cheng, K. (1986). A purely geometric module in the rat's spatial representation. Cognition, 23, 149-178.

Cheng, K. (1989). The vector sum model of pigeon landmark use. Journal of Experimental Psychology: Animal Behavior Processes, 15, 366-375.

Cheng, K. (1994). The determination of direction in landmark-based spatial search in pigeons: A further test of the vector sum model. $A n$ imal Learning \& Behavior, 22, 291-301.

Collett, T. S., Cartwright, B. A., \& Smith, B. A. (1986). Landmark learning and visuo-spatial memories in gerbils. Journal of Comparative Physiology A, 158, 835-851.

Diez-Chamizo, V., Sterio, D., \& Mackintosh, N. J. (1985). Blocking and overshadowing between intra-maze and extra-maze cues: A test of the independence of locale and guidance learning. Quarterly Journal of Experimental Psychology, 37B, 235-253.

Etienne, A. S., Berlie, J., Georgakopoulos, J., \& Maurer, R. (1998). Role of dead reckoning in navigation. In S. Healy (Ed.), Spatial representation in animals (pp. 54-68). New York: Oxford University Press.

GAllistel, C. R. (1990). The organization of learning. Cambridge, MA: MIT Press.

Greene, C. M., \& Cook, R. G. (1997). Landmark geometry and identity controls spatial navigation in rats. Animal Learning \& Behavior, 25, 312-323.

Kamil, A. C., \& Jones, J. E. (1997). The seed-storing corvid Clark's nutcracker learns geometric relationships among landmarks. Nature, 390, 276-279.

LEwIs, C. (1993). Analyzing means from repeated measures data. In G. Gideon \& L. Lewis (Eds.), A handbook for data analysis in the behavioral sciences: Statistical issues (pp. 73-89). Hillsdale, NJ: Erlbaum.

Loomis, J. M., Klatsky, R. L., Golledge, R. G., Cicinelli, J. G., PelLEGRINO, J. W., \& FrY, P. A. (1993). Nonvisual navigation by blind and sighted: Assessment of path integration ability. Journal of Experimental Psychology: General, 122, 73-91.

Morris, R. G. M. (1981). Spatial localization does not require the presence of local cues. Learning \& Motivation, 12, 239-260.

PouceT, B. (1993). Spatial cognitive maps in animals: New hypotheses on their structure and neural mechanisms. Psychological Review, 100, 163-182.

Poucet, B., Chapuis, N., Durup, M., \& Thinus-Blanc, C. (1986). A study of exploratory behavior as an index of spatial knowledge in hamsters. Animal Learning \& Behavior, 14, 93-100.

Roberts, A. D. L., \& PeArCE, J. M. (1998). Control of spatial behavior by an unstable landmark. Journal of Experimental Psychology: Animal Behavior Processes, 24, 172-184.

Roberts, W. A. (1998a). Principles of animal cognition. Boston: McGraw-Hill.

ROBERTS, W. A. (1998b, July). Spatial representation in animals and 
the use of spatial coding in the representation of other dimensions of animal cognition. Paper presented at the workshop on Spatial Schemas and Abstract Thought, Munich, Germany.

Rodrigo, T., Chamizo, V. D., McLaren, I. P. L., \& Mackintosh, N. J. (1997). Blocking in the spatial domain. Journal of Experimental Psychology: Animal Behavior Processes, 23, 110-118.

Spetch, M. L., Cheng, K., \& MaCDonald, S. E. (1996). Learning the configuration of a landmark array: I. Touch-screen studies with pigeons and humans. Journal of Comparative Psychology, 110, 55-68.

Spetch, M. L., Cheng, K., MacDonald, S. E., Linkenhoker, B. A., Kelly, D. M., \& DoERKson, S. R. (1997). Use of landmark config- uration in pigeons and humans: II. Generality across search tas Journal of Comparative Psychology, 111, 14-24.

SUTHERLAND, R. J., \& RUDY, J. W. (1989). Configural association t ory: The role of the hippocampal formation in learning, memory, : amnesia. Psychobiology, 17, 129-144.

Suzuki, S., Augerinos, G., \& Black, A. H. (1980). Stimulus cont of spatial behavior on the eight-arm maze in rats. Learning \& $M$ vation, 11, 1-18.

(Manuscript received November 2, 1998; revision accepted for publication September 17, 1999.) 